\title{
Evaluación multicriterio y multiobjetivo para optimizar la selección de áreas para establecer plantaciones forestales
}

\author{
Multi-criteria and multi-objective evaluation to optimize the selection of \\ areas to establish forest plantations
}

\author{
Pedro Hernández-Zaragoza', José René Valdez-Lazaldel* Arnulfo Aldretel y Tomás Martínez-Trinidad'
}

\begin{abstract}
Colegio de Postgraduados. Postgrado en Ciencias Forestales. Montecillo, Texcoco, Estado de México, México.
\end{abstract}

\section{RESUMEN}

La selección del sitio adecuado para establecer plantaciones forestales es crucial si se desea maximizar su probabilidad de éxito. Los análisis de aptitud de tierras sirven para ese propósito al empatar los requerimientos ecológicos de las especies a plantar con el potencial productivo del suelo y con las condiciones climáticas existentes para el crecimiento óptimo de las especies elegidas. Este estudio describe un modelo, desarrollado en el marco del proceso analítico jerárquico y la asignación de tierras multiobjetivo e integrado en un sistema de información geográfica, para seleccionar el sitio óptimo para el establecimiento de plantaciones forestales comerciales en los municipios Durango, Nombre de Dios y Súchil, del estado de Durango, México, considerando seis especies de pino (Pinus engelmannii, P. leiophylla, P. arizonica, P. cembroides, P.chibuabuana y P. durangensis). El modelo es jerárquico, considera criterios y subcriterios climáticos, edafológicos y topográficos que afectan la localización de áreas adecuadas para establecer plantaciones forestales comerciales. Los valores de los subcriterios fueron estandarizados, ponderados y ordenados mediante matrices de comparaciones pareadas, considerando su importancia para el desarrollo de plantaciones. El modelo inicialmente cuantificó la aptitud de la tierra para establecer plantaciones forestales. Posteriormente, los resultados de aptitud se optimizaron mediante un procedimiento multiobjetivo para identificar la mejor especie a plantar en la zona bajo cuatro escenarios de decisión: turno de producción, calidad de la madera, supervivencia y susceptibilidad a plagas y enfermedades. Los resultados indican que el municipio de Durango presenta la mayor cantidad de áreas óptimas para establecer plantaciones forestales con las especies analizadas.

PALABRAS ClAVE: análisis de aptitud de tierras, asignación de tierras, Durango, plantaciones comerciales de pino, proceso analítico jerárquico, toma de decisiones.

\begin{abstract}
Selecting where to establish forest plantations is crucial to maximize its probability of success. Multi-criteria decision analysis for land suitability accomplishes this purpose by matching ecological requirements of chosen species for planting against soil productive potential and climate conditions. This study describes a model developed under both a hierarchical analytical process framework and a multiobjective land allocation approach, in a GIS environment, to select optimal sites for establishing six pine species (Pinus engelmannii, $P$. leiophylla, P. arizonica, P. cembroides, P.chibuahuana and P. durangensis) for commercial purposes in Northern Mexico. The model considers climatic, edaphological and topographical criteria and subcriteria that affect the location of suitable areas to establish commercial forest plantations. Sub-criteria values were standardized, weighted and ordered by pairwise matrices considering their importance regarding to planting such tree species. Initially, the model quantified the suitability of the land to establish forest plantations. Then, such suitability results were optimized through a multiobjective procedure to identify the best species to plant in the area under four decision scenarios: harvesting age, wood quality, survival, and susceptibility to both pests and diseases. The results indicate that the municipality of Durango has the greatest number of optimal areas to establish forest plantations with the species analyzed.
\end{abstract}

KEYwORDs: land suitability analysis, land allocation, Durango, pine plantation, hierarchical analytical process, decision making. 


\section{INTRODUCCIÓN}

La demanda nacional e internacional creciente de productos forestales maderables sugiere la necesidad de complementar el esquema actual de aprovechamiento de bosques naturales con plantaciones forestales. En el año 2012 las plantaciones forestales representaron solo $7 \%$ de la superficie forestal mundial (277 millones de hectáreas), pero abastecieron 46\% del mercado global de productos maderables (Payn et al., 2015; Organización de las Naciones Unidas para la Agricultura y la Alimentación [FAO], 2016). México posee un potencial importante para el establecimiento de plantaciones forestales en diversos ecosistemas, aunque la superficie establecida es solo de aproximadamente 224000 ha, con $67 \%$ de especies maderables y $33 \%$ de especies no maderables (Comisión Nacional Forestal [Conafor], 2013).

La selección del sitio adecuado para plantar es uno de varios elementos cruciales que debe considerarse con rigor si se desea maximizar el éxito en el establecimiento de plantaciones forestales. Por ello es imperativo para este tipo de proyectos realizar un análisis de aptitud de tierras que considere los requerimientos ecológicos de las especies a plantar, y que optimice la utilización del potencial productivo de los suelos y las condiciones climáticas existentes para el crecimiento de las especies elegidas.

Existen varios enfoques de evaluación para este propósito. Entre los más conocidos están el esquema FAO (1993), el álgebra Booleana (Delgado-Caballero, ValdezLazalde, Fierros-González, de los Santos-Posadas y Gómez-Guerrero, 2010) y la evaluación multicriterio en varias modalidades (Gómez y Barredo, 2005; BustillosHerrera, Valdez-Lazalde, Aldrete y González-Guillén, 2007). La elección del enfoque y método adecuados depende de los objetivos específicos de la plantación. Sin embargo, independiente del método a utilizar, las técnicas de evaluación multicriterio y multiobjetivo, en el marco de un sistema de información geográfica (SIG), han demostrado ser herramientas de mayor útilidad para la evaluación, el análisis y el mapeo de la aptitud de tierras para propósitos predefinidos, al reducir la subjetividad, siempre presente en este tipo de procesos (Malczewski, 2004; Santé-
Riveira y Crecente-Maseda, 2005), además de permitir el analisis conjunto de información del ambiente y la referente a la experiencia de los tomadores de decisiones (Saaty, 1990; Delgado-Caballero et al., 2010). En México, particularmente en el sector forestal, poco se han utilizado estas técnicas incorporadas en un SIG, y no van más allá del análisis multicriterio realizado con enfoque Booleano (MartínezSalvador y Prieto-Ruiz, 2011) o mediante procedimientos de mayor complejidad como el proceso analítico jerarquizado (AHP), la lógica fuzzy, entre otros (BustillosHerrera et al., 2007; Olivas-Gallegos, Valdez-Lazalde, Aldrete, González-Guillén y Vera-Castillo, 2007; DelgadoCaballero et al., 2010; Silva-Gallegos et al., 2016; GonzálezOvando, Plascencia-Escalante y Martínez-Trinidad, 2016; Aguirre-Salado et al., 2017) que se enfocan en un objetivo único. Solo el trabajo de Aguirre-Salado, Valdez-Lazalde, Sánchez-Díaz, Miranda-Aragón y Aguirre-Salado (2015) aborda el enfoque de análisis multiobjetivo.

\section{OBJETIVOS}

El estudio se planteó con dos objetivos paralelos, por un lado, identificar en el marco de un proceso de toma de decisiones multicriterio y multiobjetivo espacial, estructurado conforme al proceso analítico jerárquico (AHP, por sus siglas en inglés Saaty, 1980; Saaty, 1990; Brunelli, 2015), las áreas con la mejor aptitud para el establecimiento de plantaciones forestales comerciales con seis especies de pino (Pinus durangensis, P. engelmannii, P. arizonica, $P$. chibuabuana, $P$. leiophylla y $P$. cembroides), que compiten por el espacio más adecuado en los municipios de Durango, Nombre de Dios y Súchil del estado de Durango, México. A excepción de P. cembroides, estas especies son las más importantes en cuanto a abundancia y calidad de madera en la región bajo análisis (García-Arévalo y González-Elizondo, 2003). Por otro lado, el estudio pretende presentar una propuesta detallada de modelo geoespacial para realizar de manera transparente evaluaciones de aptitud de tierras para establecer plantaciones forestales comerciales. 


\section{MATERIALES Y MÉTODOS}

\section{Área de estudio}

La investigación se desarrolló en los municipios de Durango, Nombre de Dios y Súchil del estado de Durango, México (Fig. 1), localizados entre las coordenadas 23 $13^{\circ}$ y $24^{\circ} 43^{\prime} \mathrm{N}$ y $103^{\circ} 79^{\prime}$ y $105^{\circ} 58^{\prime} \mathrm{O}$, con una superficie aproximada de $12342 \mathrm{~km}^{2}$.

Cartografía. Considerando el marco metodológico del AHP definido por Saaty $(1980,1990)$ se integró una base de datos, correspondiente al área de estudio, que describe la variación espacial de los criterios y los subcriterios previamente identificados como los más importantes para el establecimiento de plantaciones con las especies de interés (Fig. 2). La información disponible de estos criterios se homogeneizó en cuanto a proyección cartográfica y datum geodésico para generar el modelado cartográfico a escala 1:250 000.
Los mapas de temperatura máxima, mínima y precipitación anual, se recortaron de las cartas temáticas escala 1:250 000 de la Comisión Nacional para el Conocimiento y Uso de la Biodiversidad (Conabio). Los mapas edafológicos del Instituto Nacional de Estadística, y Geografía (Inegi) se digitalizaron a escala 1:50 $000 \mathrm{y}$ a partir de estos se generaron mapas de textura y profundidad del suelo; esta última se calculó al asociar una profundidad con la fase física observada. Suelos con fase física lítica, gravosa o pedregosa se les asignó una profundidad de $0.1 \mathrm{~m}$ a $1 \mathrm{~m}$; suelos sin fase física, se consideraron de profundidad $>1 \mathrm{~m}$. El mapa de $\mathrm{pH}$ del suelo se obtuvo de la cartografía derivada de la actualización del Inventario Nacional Forestal y de Suelos 2000-2006, realizado por la Secretaría del Medio Ambiente y Recursos Naturales (Semarnat) y el Colegio de Postgraduados (CP), escala 1:250 000 (Semarnat-CP, 2002). El modelo digital de elevaciones se obtuvo del sistema de elevación digital para México (Inegi, 2005) con puntos de muestreo de $1 \mathrm{~s} \times 1 \mathrm{~s}$ de arco; del mismo modelo se derivó la pendiente.

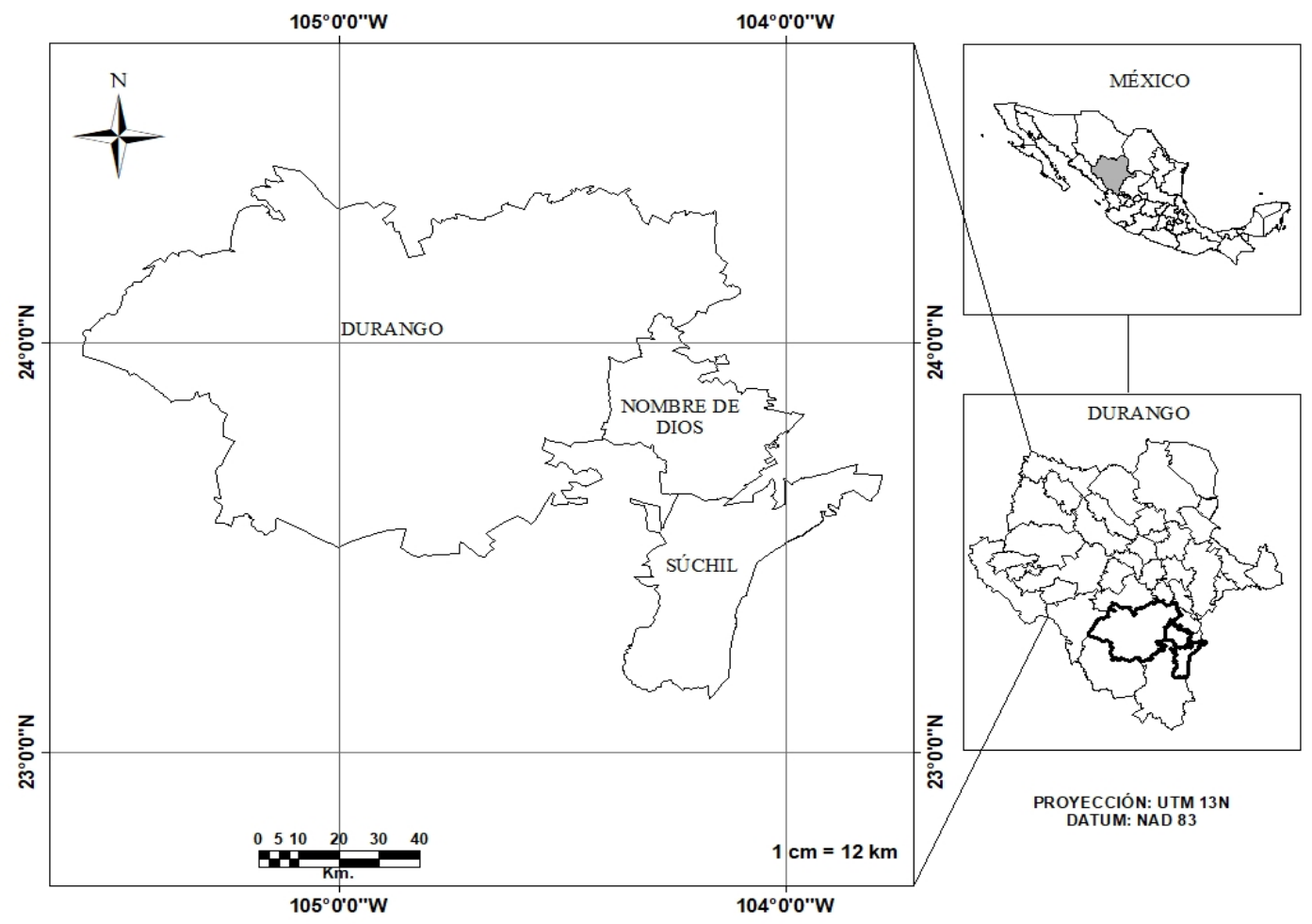

Figura 1. Localización del área de estudio en el estado de Durango, México. 


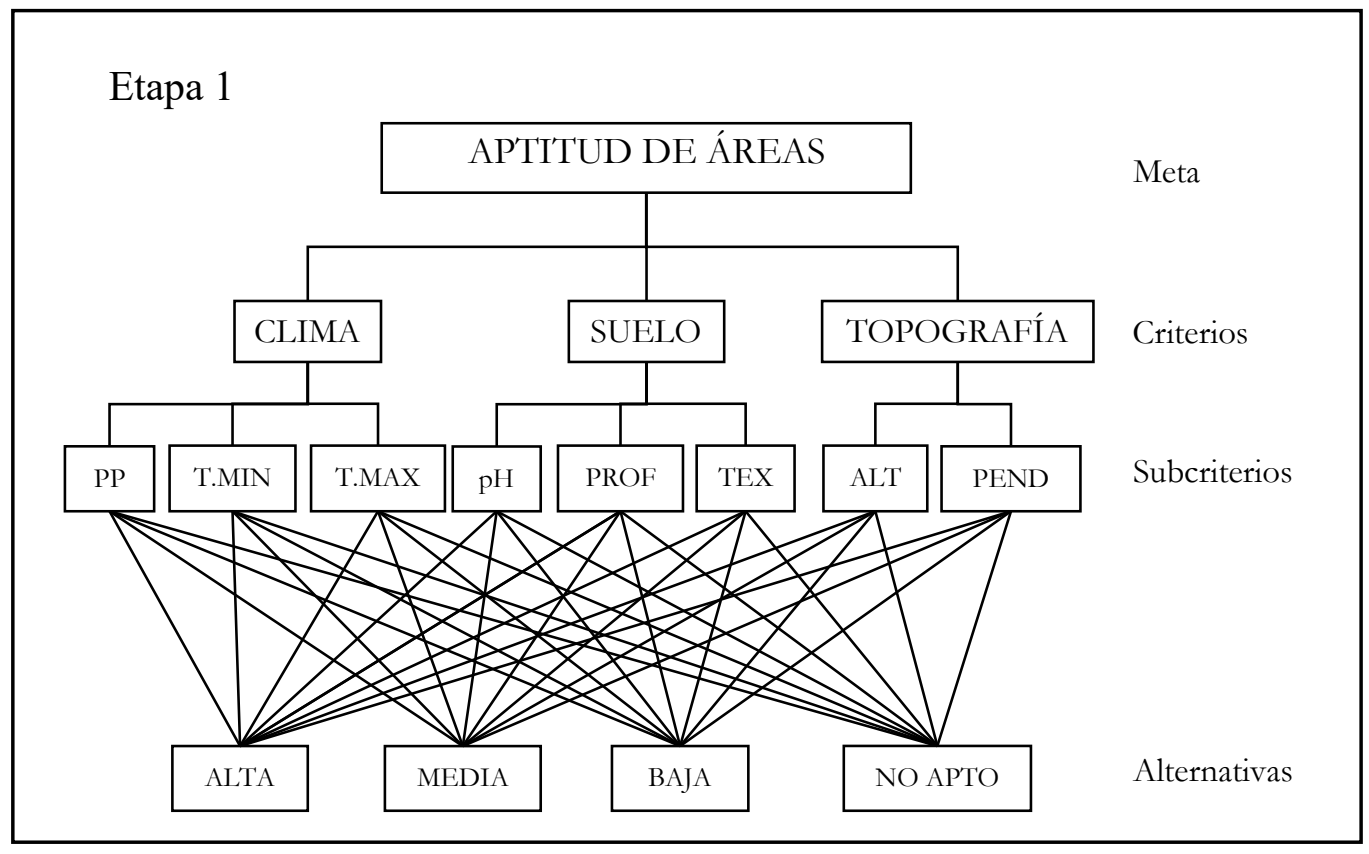

FIGURA 2. Jerarquización de los criterios y subcriterios del problema de decisión.

$\mathrm{PP}=$ precipitación total anual; $\mathrm{T} . \mathrm{MIN}=$ temperatura mínima; $\mathrm{T} . \mathrm{MAX}=$ temperatura máxima; $\mathrm{PROF}=$ profundidad; TEX = textura; $\mathrm{ALT}=$ altitud; PEND = pendiente.

\section{Procedimiento}

El estudio se desarrolló en dos etapas: Etapa 1, corresponde a la realización de un análisis espacial multicriterio (realización específica del AHP) para definir la aptitud del área de estudio para cada una de las seis especies de interés; Etapa 2, corresponde a un análisis espacial multiobjetivo (MOLA -Eastman, Jin, Kyem y Toledano, 1995; Eastman, 2012), llevado a cabo para identificar la especie más adecuada para plantar en la zona. Para robustecer el análisis, se generaron cuatro escenarios de decisión con base en la aplicación de encuestas a cinco profesionales residentes en el estado de Durango, con experiencia en el establecimiento de plantaciones. Los párrafos siguientes detallan lo realizado en cada etapa.

\section{Etapa 1. Evaluación espacial multicriterio mediante}

AHP. Esta etapa permitió identificar las áreas con aptitud para el establecimiento de plantaciones forestales comerciales con las seis especies de interés $(P$. durangensis, $P$. engelmannii, $P$. arizonica, $P$. chibuabuana, $P$. leiophylla y $P$. cembroides), considerando sus requerimientos climáticos, edafológicos y topográficos. Con base en información obtenida de literatura científica sintetizada en ValdezLazalde, López-Upton y Aldrete (2003) y en la opinión recabada de 11 expertos en plantaciones forestales comerciales en México (Delgado-Caballero et al., 2010), se identificaron los criterios y subcriterios importantes (variables o requerimientos ecológicos) para el buen desarrollo de las seis especies de interés: clima (temperatura mínima, temperatura máxima y precipitación anual), suelo (profundidad, textura y $\mathrm{pH}$ ) y topografía (altitud y pendiente).

Los niveles de aptitud de la tierra para cada especie se definieron a través de la realización del AHP (Saaty, 1980, 1990), un procedimiento matemático para la medición y toma de decisiones multicriterio, utilizando como regla de decisión a la denominada combinación lineal ponderada (WLC) (Malczewski, 2004; Gómez y Barredo, 2005; Malczewski, 2011). La estructura jerárquica de los criterios y subcriterios utilizados se presenta en la figura 2 .

Estandarización de subcriterios. El AHP requiere que los datos y la información útil para el proceso estén definidos en una escala de medición común, para ello fue necesario estandarizar los mapas utilizados (subcriterios). 
Se asignaron valores entre 0 y 1 para cada nivel de subcriterios en función de los requerimientos ecológicos de las especies mediante el método de comparaciones pareadas (Saaty, 1980; Olivas-Gallegos et al., 2007). A continuación, se ejemplifica el procedimiento para estandarizar el subcriterio precipitación teniendo como meta plantar $P$. duranguensis (Supl. 1, Tabla S1). Inicialmente se definieron cinco niveles o clases de aptitud para el subcriterio considerando los valores originales de precipitación $(\mathrm{mm})$ presentes en el área de estudio: S1 (1000 - 1800), S2 (800 1000), S3 (600 - 800), N1 (500 - 600) y N2 (400 - 500); donde S1 representa el cumplimiento adecuado del subcriterio para $P$. duranguensis, cumplimiento que decrece hasta N2, que corresponde al nivel más bajo de aptitud o aquel de menor cumplimiento del subcriterio. Las cinco clases se definieron buscando facilitar la compresión y análisis del proceso de estandarización. Posteriormente, las clases de aptitud se colocaron en una matriz pareada (Tabla 1) y se usó la escala de comparación definida por Saaty (1980, 1990), de 1/9 a 9, para estandarizarlas mediante el cálculo de máximo eigen vector (columna VE, Tabla 1). Los detalles del método pueden consultarse en Malczewsky (1999, p. 182). Mediante procedimiento similar fueron estandarizados el resto de los subcriterios utilizados en el estudio (Supl. 1, Tablas S1-S6).

TABLA 1. Matriz de comparaciones pareadas y valor estandarizado del subcriterio precipitación en el análisis de aptitud de Pinus durangensis.

\begin{tabular}{ccccccc}
\hline NA Precipitación & $S 1$ & $S 2$ & $S 3$ & $N 1$ & $N 2$ & $V E$ \\
\hline$S 1$ & 1 & 3 & 5 & 8 & 9 & 1 \\
$S 2$ & $1 / 3$ & 1 & 4 & 7 & 8 & 0.586 \\
$S 3$ & $1 / 5$ & $1 / 3$ & 1 & 6 & 7 & 0.300 \\
$\mathrm{~N} 1$ & $1 / 8$ & $1 / 7$ & $1 / 6$ & 1 & 3 & 0.095 \\
$\mathrm{~N} 2$ & $1 / 9$ & $1 / 8$ & $1 / 7$ & $1 / 3$ & 1 & 0.057 \\
\hline
\end{tabular}

NA = nivel de aptitud; $V E$ = valor estandarizado de clase de subcriterio
Ponderación de criterios y subcriterios (W). La importancia relativa (peso) de los criterios y subcriterios identificados como relevantes se determinó utilizando también la metodología de comparaciones pareadas de Saaty (1980). Esta consistió en procesar información proveniente de 11 encuestas aplicadas a igual número de expertos en el establecimiento de plantaciones forestales (Delgado-Caballero et al., 2010). Cada uno de los entrevistados completó matrices de comparación pareada en un intento de jerarquizar, a su juicio y con base en su conocimiento, la importancia de los criterios y subcriterios. Las matrices resultantes se evaluaron con ayuda del software IDRISI ${ }^{\circledR}$ Selva a través del módulo GIS Analysis/Decision Support/WEIGHT (Eastman, 2012; Delgado-Caballero et al., 2010). A partir de los juicios consistentes de los expertos y mediante el cálculo de la media aritmética de los juicios se obtuvieron los valores de ponderación para ser asignados a los criterios y subcriterios en cada jerarquía.

Aplicación del proceso análitico jerárquico (AHP) y de la combinación lineal ponderada (WLC). AHP divide un problema complejo de decisión, en grupos o jerarquías simples mediante la construcción de un modelo de tres niveles: definición de la meta u objetivo, identificación y definición de criterios para evaluación de alternativas e identificación de alternativas de decisión (Fig. 2). En el programa IDRISI ${ }^{\circledR}$, a través del módulo Modeling/Macro modeler, se construyó un modelo cartográfico que permitió aplicar el modelo desarrollado en el marco del AHP, utilizando la WLC como regla de decisión para sintetizar y ordenar los resultados. El algoritmo consiste en: 1) los mapas de subcriterios estandarizados se multiplicaron por el peso (W) definido por los expertos para obtener los mapas de subcriterios ponderados, 2) los mapas ponderados se sumaron y dieron como resultado los mapas de criterios (clima, suelo y topografía), 3) cada mapa de criterio se multiplicó por el peso definido por los expertos para cada criterio, 4) los mapas que contienen los criterios se sumaron para obtener el mapa de aptitud preliminar (Fig. 3, Olivas-Gallegos et al., 2007). 


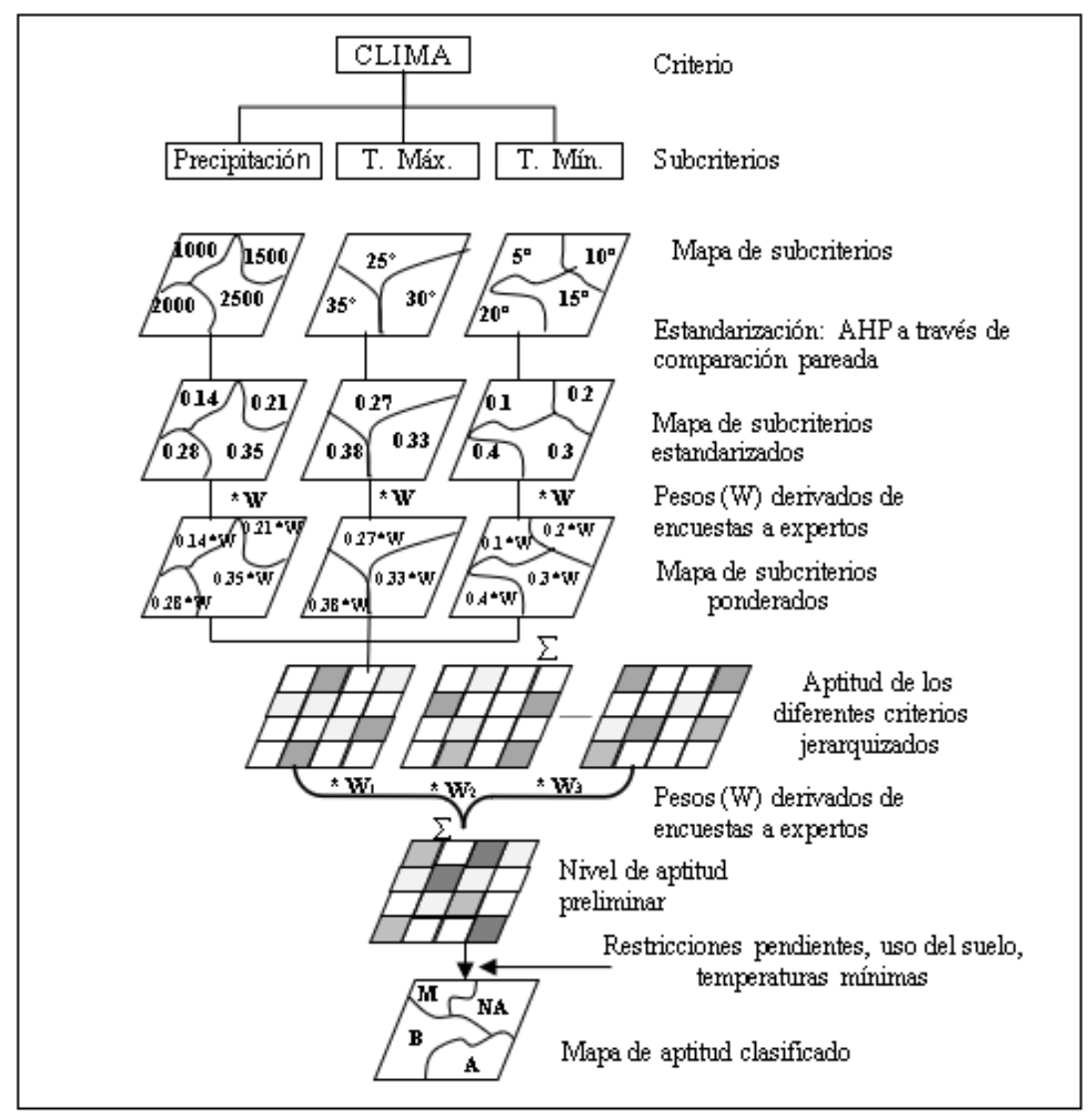

FIGURA 3. Proceso de análisis jerarquizado realizado para definir áreas con aptitud para plantar cada una de las especies (modificado de Olivas-Gallegos et al., 2007).

En la última fase de la Etapa 1, el mapa de aptitud preliminar se multiplicó por un mapa binario para discriminar las áreas con restricción para el desarrollo de plantaciones (áreas forestales arboladas, áreas de conservación biológica), el uso permanente definido (urbano, carreteras, cuerpos de agua), áreas de mayor rentabilidad (agricultura intensiva) y áreas con pendientes mayores a 30\%. Posteriormente, para facilitar la interpretación, los mapas resultantes de la aplicación del modelo cartográfico para cada especie se clasificaron en cuatro clases o niveles de aptitud: valores de 0.9 a 1 se etiquetaron como clase de aptitud alta, valores de 0.8 a 0.9 como clase de aptitud media, valores de 0.7 a 0.8 como aptitud baja y finalmente, valores de 0 a 0.7 como clase no apta (aptitud marginal). La clasificación propuesta responde a la necesidad de identificar con claridad las áreas de mayor aptitud. Las áreas de aptitud marginal son de interés limitado para el tomador de decisiones, por ello se agruparon en un intervalo amplio (0 a 0.7).

Etapa 2. Evaluación espacial multiobjetivo. Esta etapa permitió identificar y resolver conflictos de aptitud entre las especies consideradas, además de asignar el sitio óptimo de plantación para cada especie. Una vez elaborados los mapas de aptitud para cada una de las seis especies, se realizó una intersección espacial de estos con el objetivo de identificar conflictos en la asignación de tierras. En términos genéricos, los conflictos se resolvieron mediante una solución compromiso, específicamente mediante la técnica denominada asignación de tierras multiobjetivo (MOLA, por sus siglas en inglés -Eastman et al., 1995; Eastman, 2012). Para ello fue necesario estimar el peso relativo (ponderación) de cada especie, esto es, el nivel de preferencia de una especie sobre otra (s) y definir una meta de superficie a plantar con cada especie (Fig. 4). 


\section{Ponderación de especies y escenarios de decisión. Con} la finalidad de ponderar la importancia de las especies a plantar, se aplicaron encuestas a cinco profesionales forestales con experiencia en el establecimiento de plantaciones forestales y residentes en el área de estudio. Se les plantearon cuatro escenarios de decisión con base en las características de cada especie respecto a: la extensión del periodo de producción, la calidad de la madera, la supervivencia y la susceptibilidad a plagas y enfermedades.
Para cada escenario se pidió a los expertos indicar, mediante una matriz de comparaciones pareadas (Saaty, 1980; OlivasGallegos et al., 2007), su preferencia de una especie sobre las demás. Los datos obtenidos se procesaron con la ayuda del módulo GIS Analysis/Decision Support/WEIGHT del software IDRISI ${ }^{\circledR}$. Únicamente se consideraron los resultados que mostraron un valor de consistencia menor a 0.10 (Saaty, 1980), después se obtuvo el promedio de las ponderaciones de cada experto.

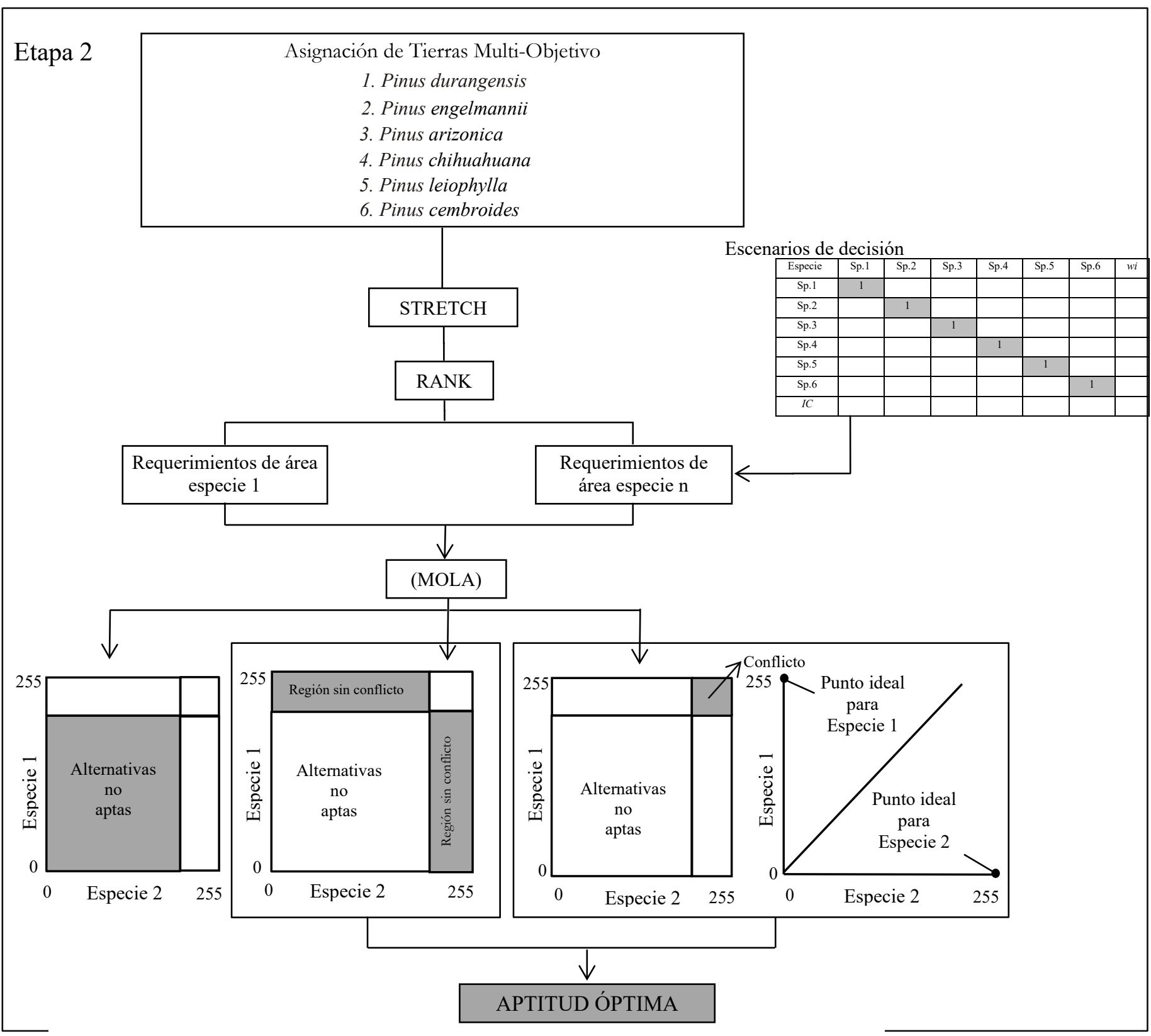

FIgURA 4. Procedimiento de asignación de tierras multiobjetivo (MOLA) en la Etapa 2 del análisis. 
Resolución de conflictos. Los mapas de aptitud de las seis especies de pino se reclasificaron a un intervalo de valores de 0 a 255, donde 0 es aptitud nula y 255 aptitud máxima. Este cambio de escala fue necesario para utilizar los módulos STRETCH y RANK de IDRISI ${ }^{\circledR}$ (Eastman, 2012). Posteriormente se asignaron los pesos definidos por los expertos a cada especie dentro de cada escenario de decisión y se definió una meta de superficie a plantar para cada especie; para ello se consideraron los umbrales de superficie existente en el área de estudio con nivel de aptitud alta para cada especie. En proyectos reales la superficie meta a plantar puede definirse considerando los objetivos de producción del proyecto y la disponibilidad de recursos para su ejecución.

Áreas donde la aptitud es alta para dos o más especies se califica como zona de conflicto. Para resolverlo, el algoritmo MOLA reclasifica iterativamente los mapas de aptitud realizando una primera asignación para cada especie de interés, busca las zonas de conflicto y las reasigna a una especie basándose en la regla de distancia mínima al punto ideal multiobjetivo empleando los valores de ponderación para las especies (Eastman et al., 1995; Eastman, 2012). La figura 4 ilustra el procedimiento en términos simples, considerando dos especies con áreas en conflicto: Especie 1 y Especie 2. En el análisis realizado para este estudio, el espacio de decisión fue de seis dimensiones (especies), lo que significa que los conflictos se resolvieron en un espacio 6-D. El procedimiento se llevó a cabo en el módulo GIS Analysis/Decision Support/MOLA de IDRISI ${ }^{\circledR}$ (Eastman, 2012).

\section{RESULTADOS Y DISCUSIÓN}

Para los expertos forestales consultados, el criterio más importante para definir el establecimiento de una plantación fue el clima, con un valor ponderado de 0.54, seguido por el suelo 0.35 y finalmente la topografía con valor de 0.11 . La tabla 2 concentra estos valores, además de los pesos asignados a los subcriterios considerados en la jerarquización del problema.

Los mapas de aptitud para plantaciones de pino elaborados mediante el proceso de evaluación espacial multicriterio se muestran en la figura 5. La comparación visual de estos mapas evidencia que algunas áreas muestran aptitud para más de una especie, lo que implica conflicto y la necesidad de sopesar la decisión respecto a cuál de las especies consideradas se debe plantar. Destaca también que el municipio de Durango posee la mayor superficie con aptitud alta para el establecimiento de plantaciones con cualquiera de las especies consideradas (Tabla 3). Superficies menores revelan aptitud media o baja en el municipio de Súchil y solamente dos especies de Pinus presentan aptitud baja en un área reducida del municipio de Nombre de Dios. Estos resultados son acordes a lo esperado dada la distribución espacial de los criterios considerados para el adecuado desarrollo de las especies de pino, particularmente la precipitación (el criterio más relevante de todos los considerados (Tabla 2), la cual es relativamente baja en los municipio de Nombre de Dios y Súchil.

La figura 6 muestra las áreas en conflicto para las especies, es decir, los terrenos que presentan aptitud adecuada (alta) para plantar dos o más especies. En estas áreas las especies "compiten" por el espacio más adecuado para su desarrollo. Dicho de otra manera, el administrador (tomador de decisiones) debe elegir entre plantar una u otra especie en función de criterios más allá de la aptitud del terreno.

La distribución espacial de las zonas óptimas para cada especie de Pinus, obtenida con base en el punto ideal multiobjetivo (MOLA), para cada uno de los cuatro escenarios identificados se ilustra en las figuras 7, 8, 9 y 10. En el escenario 1, que considera pesos diferentes, de acuerdo con la opinión de los expertos para cada especie en función de la "extensión del periodo de producción" (donde turno más corto es más deseable, Fig. 7), $P$. engelmannii podría ser plantado en $31 \%$ de la superficie disponible para el establecimiento de plantaciones forestales comerciales, seguido por $P$. arizonica $(22.5 \%)$ y $P$. durangensis $(16.5 \%)$. Contrariamente, $P$. leiophylla, $P$. chibuabuana y $P$. cembroides resultaron las especies con menor superficie apta. Lo anterior se aprecia mejor en el concentrado de datos de la tabla 4. 
Madera y Bosques vol. 25, núm. 2, e2521819 Verano 2019

TABLA 2. Ponderación asignada por los expertos a los criterios y subcriterios considerados en el establecimiento de plantaciones forestales.

\begin{tabular}{lccccc}
\hline \multicolumn{5}{c}{ Criterios } \\
\hline Criterio & Peso (W) & Criterio & Peso (W) & Criterio & Peso (W) \\
Clima & 0.5389 & Suelo & 0.3554 & Topografía & O.1057 \\
& \multicolumn{5}{c}{ Subcriterios } \\
Clima & Peso (W) & Suelo & Peso (W) & Topografía & Peso (W) \\
Precipitación & 0.6251 & Profundidad & 0.4333 & Altitud & 0.5682 \\
T. Máxima & 0.1710 & Textura & 0.2437 & Pendiente & 0.4318 \\
T. Mínima & 0.2039 & $\mathrm{pH}$ & 0.3230 & & - \\
\hline
\end{tabular}

TABLA 3. Superficies (ha) con aptitud para las especies de interés por municipio.

\begin{tabular}{|c|c|c|c|c|c|}
\hline \multirow[b]{2}{*}{ Especie } & \multirow[b]{2}{*}{ Aptitud } & \multicolumn{3}{|c|}{ Municipio } & \multirow[b]{2}{*}{ Total (ha } \\
\hline & & Durango & N. de Dios & Súchil & \\
\hline \multirow{3}{*}{ P. durangensis } & alta & 6464 & 0 & 0 & 6464 \\
\hline & media & 3908 & $\mathrm{O}$ & $\mathrm{O}$ & 3908 \\
\hline & baja & 13353 & $\mathrm{O}$ & 0 & 13353 \\
\hline \multirow{3}{*}{ P. engelmannii } & alta & 8298 & $\mathrm{O}$ & $\mathrm{O}$ & 8298 \\
\hline & media & 1441 & 0 & 0 & 1441 \\
\hline & baja & 6450 & $\mathrm{O}$ & 10159 & 16609 \\
\hline \multirow{3}{*}{ P. arizonica } & alta & 12483 & $\mathrm{O}$ & 0 & 12483 \\
\hline & media & 14500 & $\mathrm{O}$ & 589 & 15089 \\
\hline & baja & 40209 & 2065 & 15829 & 58104 \\
\hline \multirow{3}{*}{ P. chihuahuana } & alta & 9142 & $\mathrm{O}$ & 0 & 9142 \\
\hline & media & 13761 & 0 & 0 & 13761 \\
\hline & baja & 48161 & $\mathrm{O}$ & 0 & 48161 \\
\hline \multirow{3}{*}{ P. leiophylla } & alta & 8149 & 0 & $\mathrm{O}$ & 8149 \\
\hline & media & 4394 & $\mathrm{O}$ & $\mathrm{O}$ & 4394 \\
\hline & baja & 9707 & $\mathrm{O}$ & 0 & 9707 \\
\hline \multirow{3}{*}{ P. cembroides } & alta & 27508 & $\mathrm{O}$ & 0 & 27508 \\
\hline & media & 50964 & $\mathrm{O}$ & 12871 & 63835 \\
\hline & baja & 57375 & 2096 & 9498 & 68969 \\
\hline
\end{tabular}




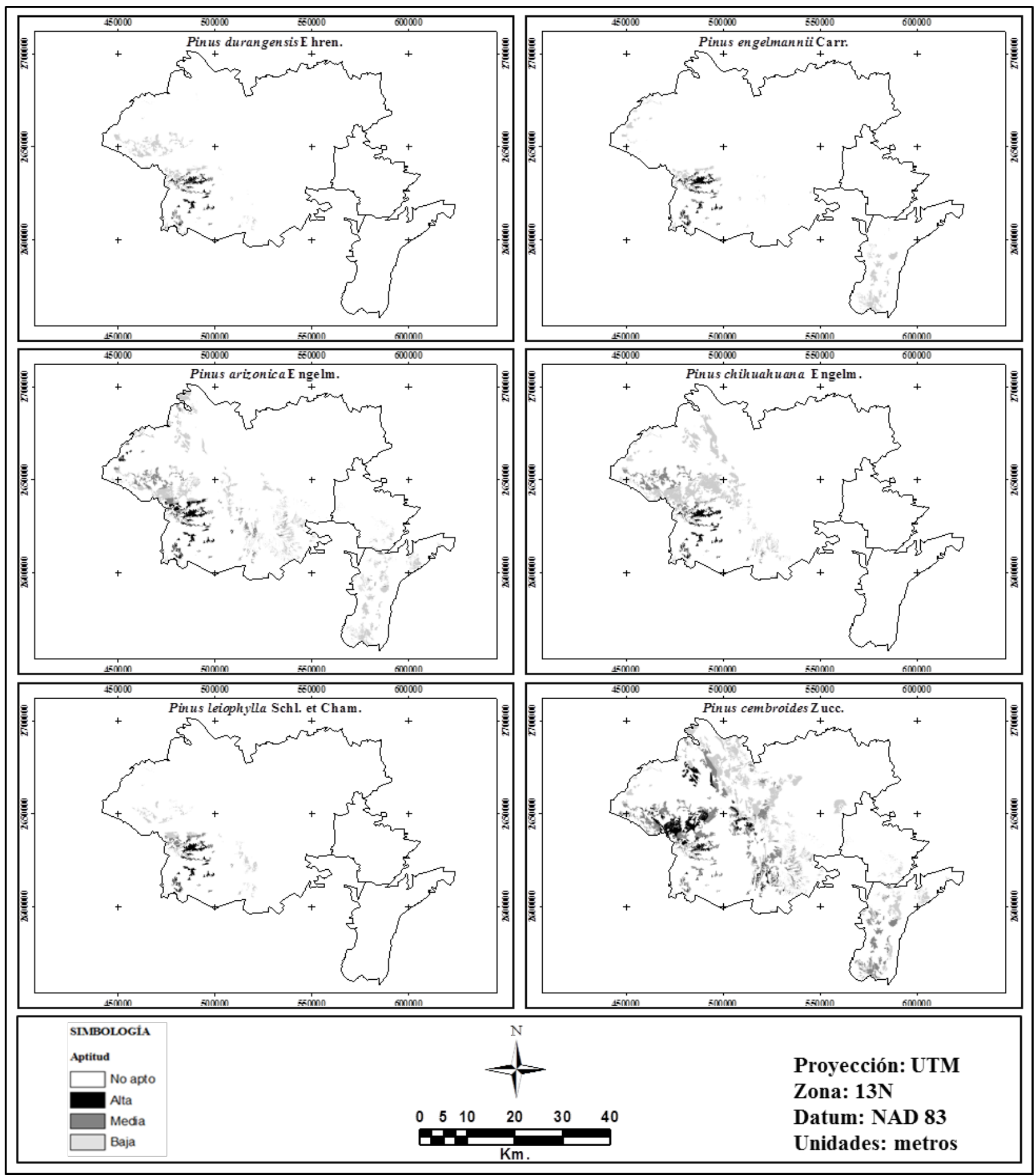

FIGURA 5. Localización de áreas con aptitud para seis especies de Pinus en tres municipios del estado de Durango. 


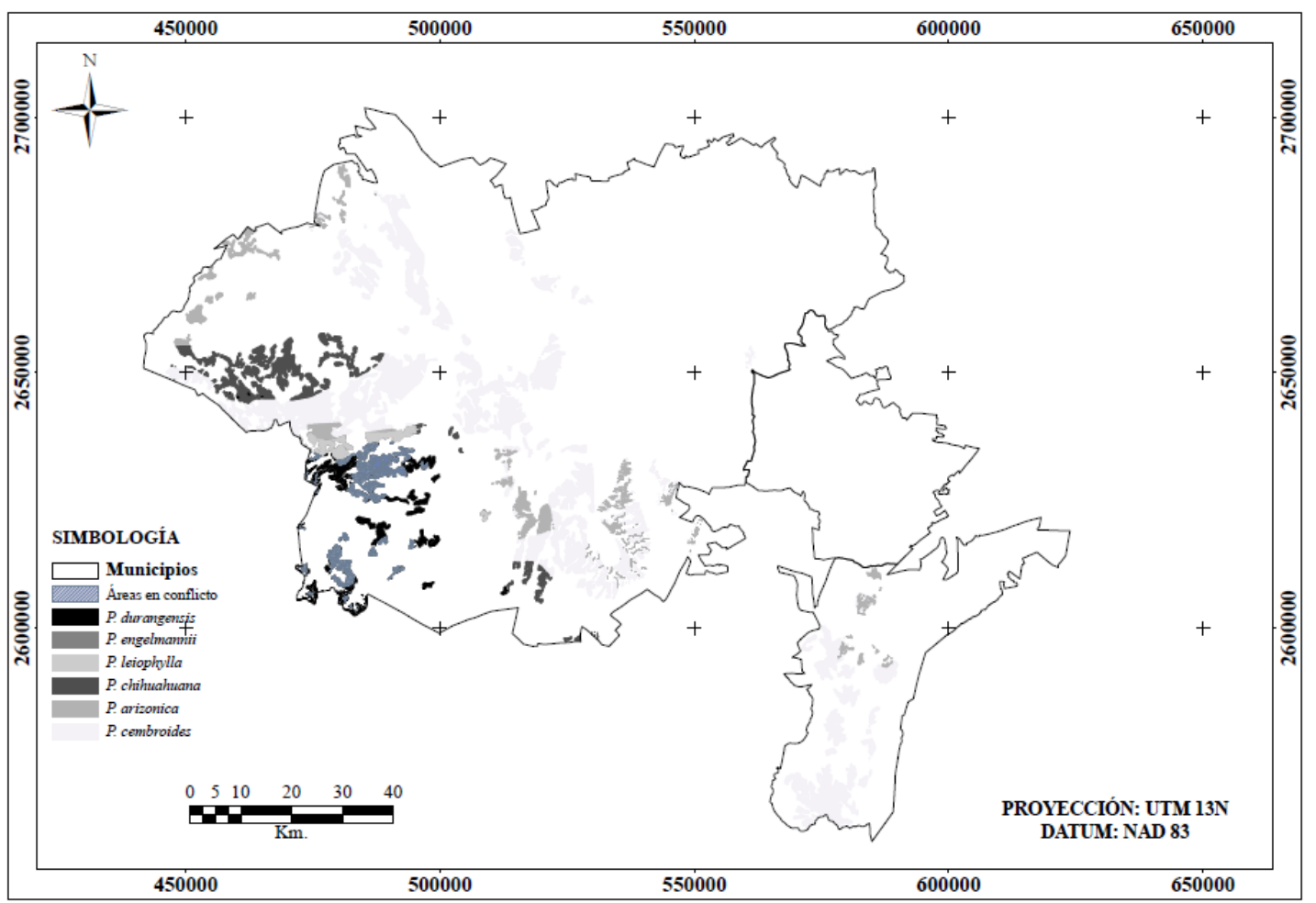

FIgURA 6. Localización de áreas en conflicto para las seis especies de Pinus en tres municipios del estado de Durango.

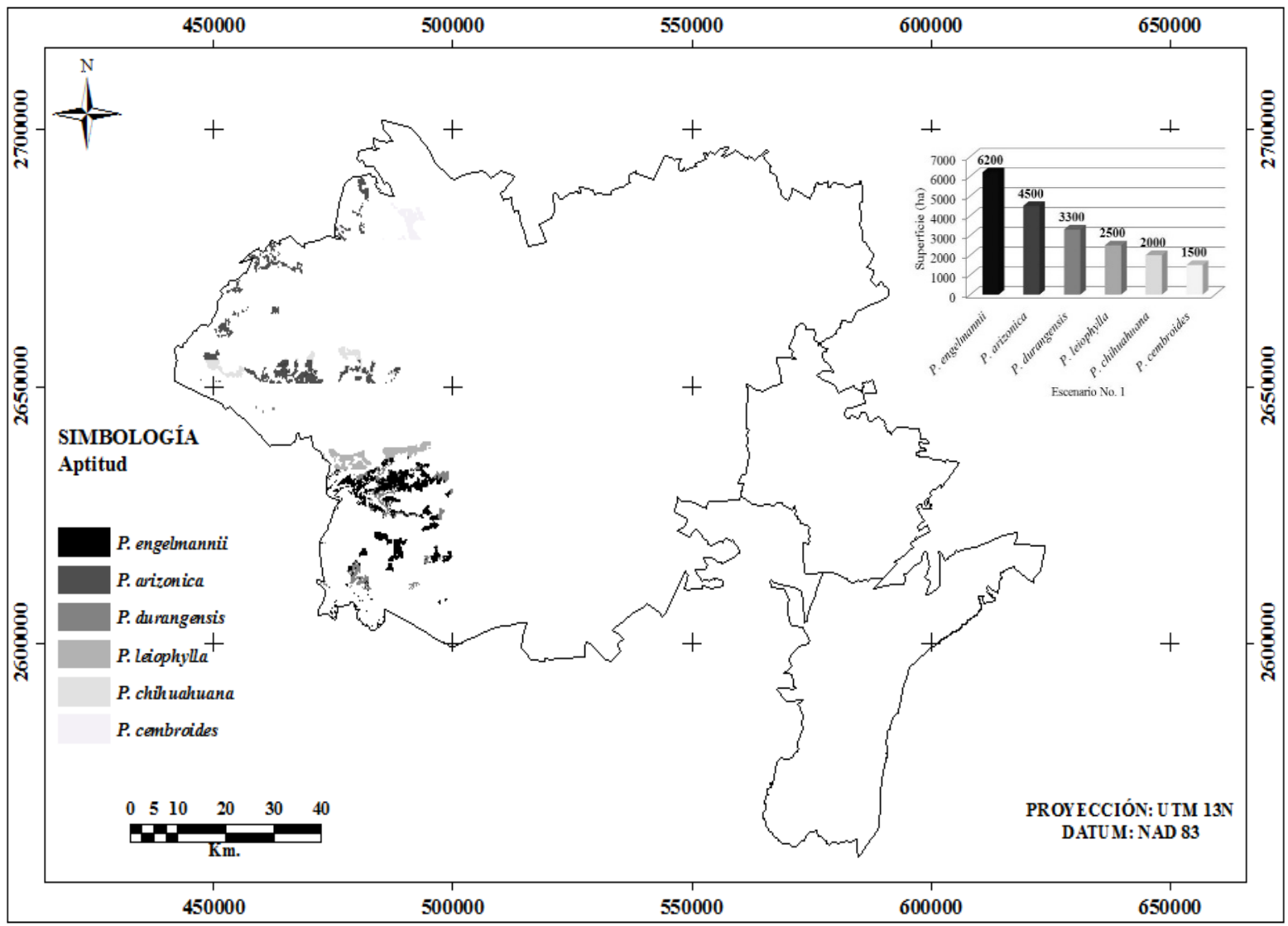

FIGURA 7. Distribución espacial óptima para cada especie de Pinus, considerando el escenario "extensión del periodo de producción". 


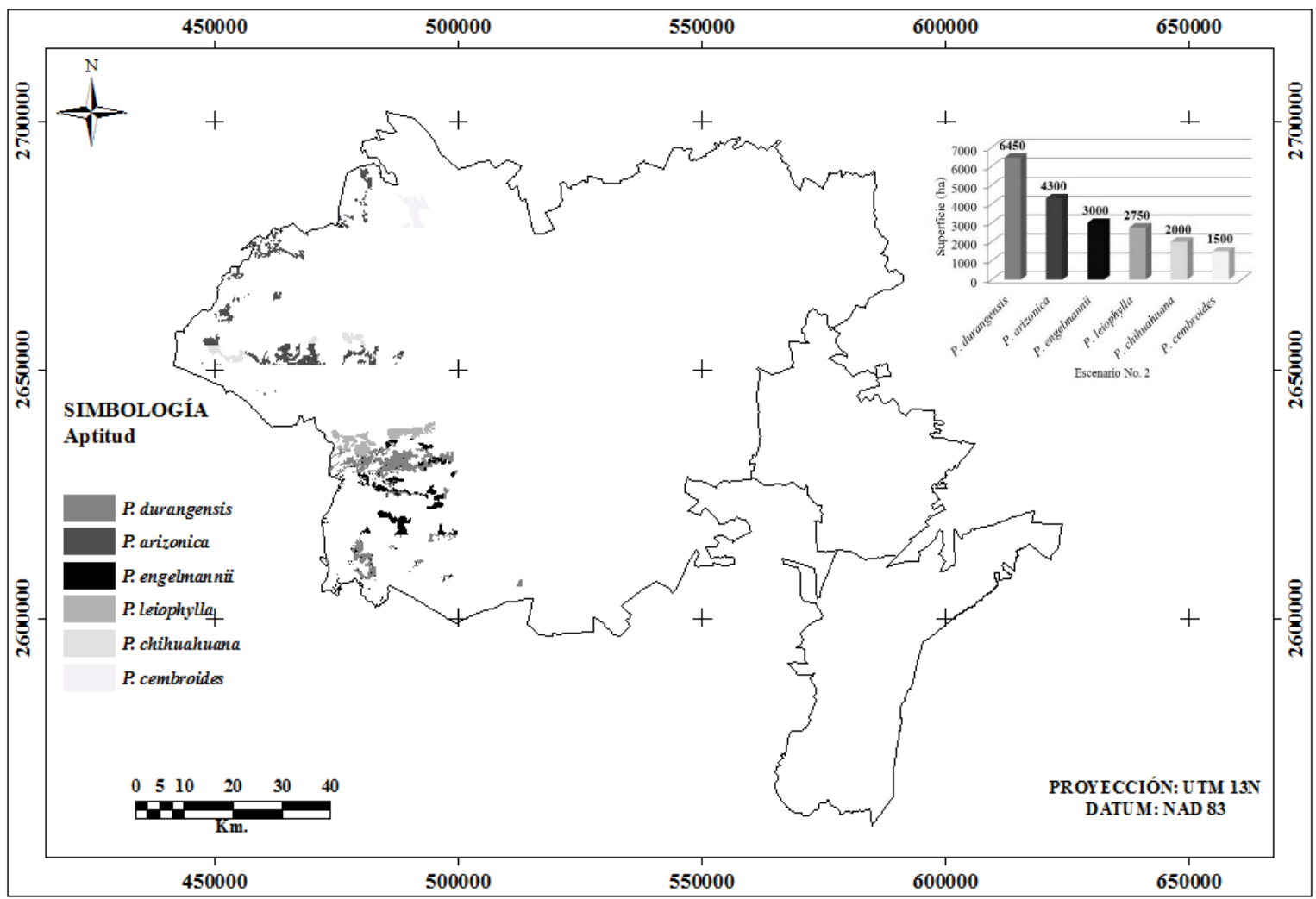

FIGURA 8. Distribución espacial óptima para cada especie de Pinus, considerando el escenario "calidad de la madera".

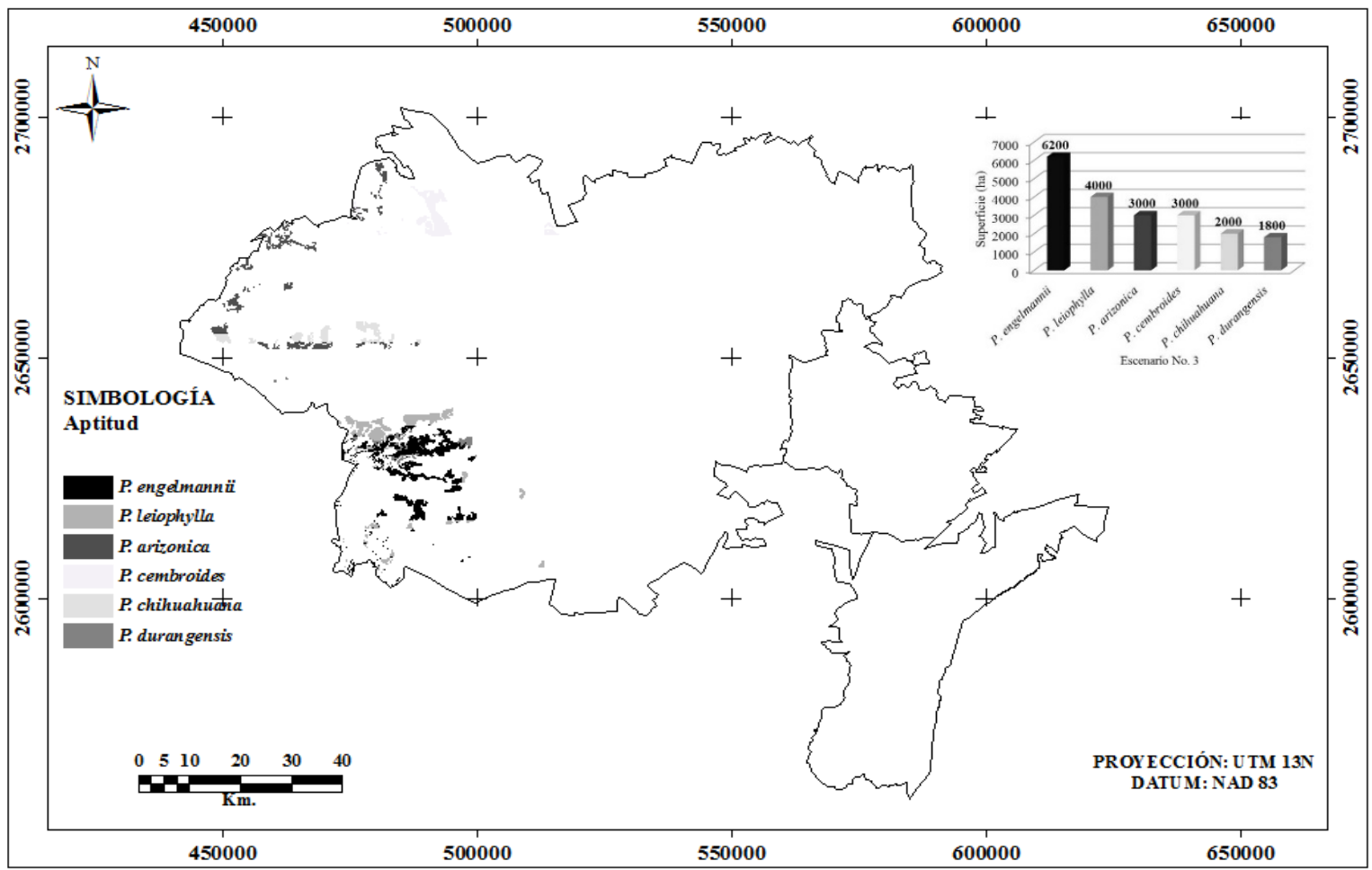

FIGURA 9. Distribución espacial óptima para cada especie de Pinus, considerando el escenario "supervivencia de la especie". 


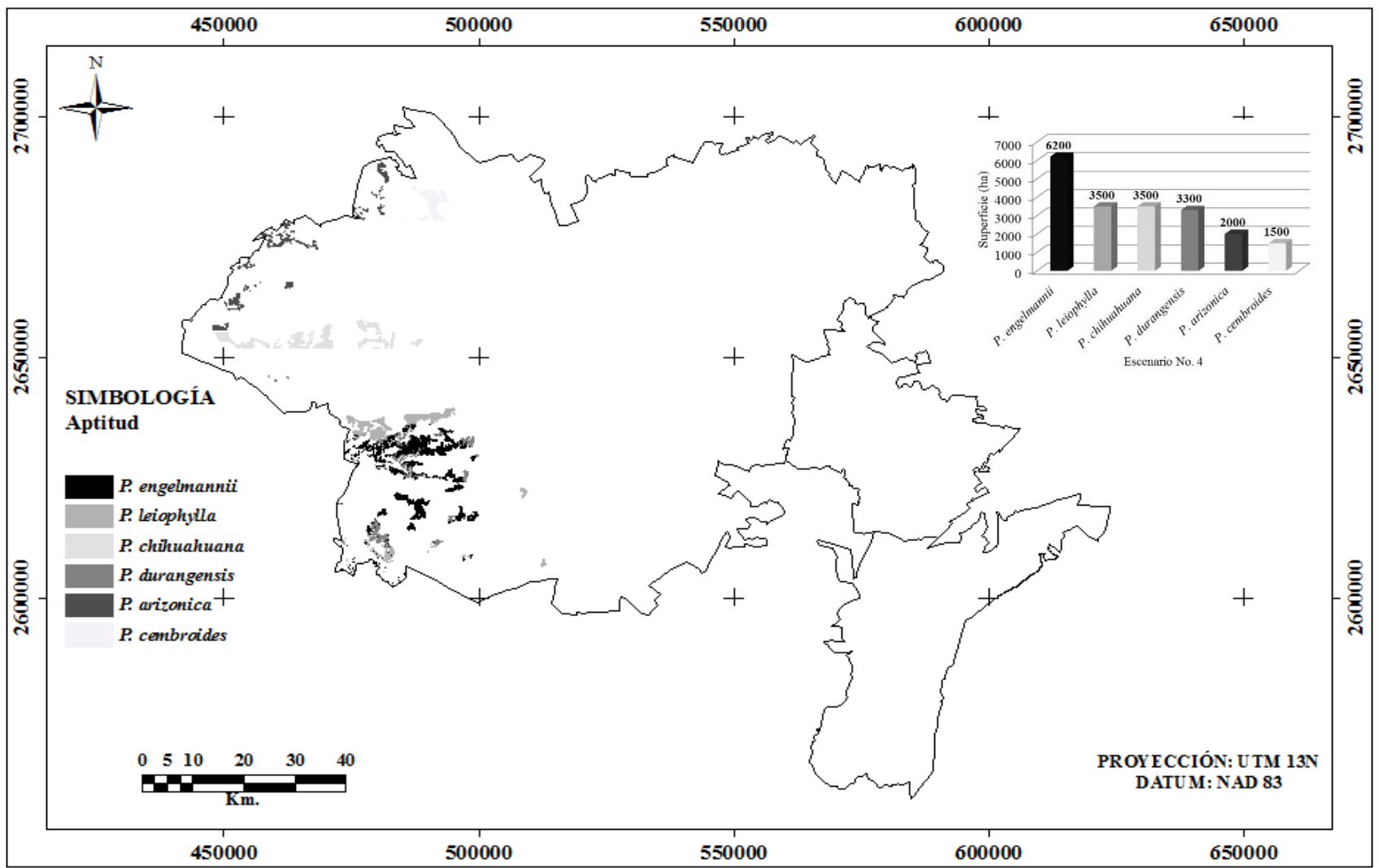

FIGURA 10. Distribución espacial óptima para cada especie de Pinus, considerando el escenario "susceptibilidad a plagas y enfermedades".

TABLA 4. Ponderación asignada, superficie de plantación y \% de la superficie total para cada especie y escenarios considerados.

\begin{tabular}{|c|c|c|c|c|c|c|c|c|c|c|c|c|}
\hline \multirow{3}{*}{ Especie } & \multicolumn{12}{|c|}{ Escenarios } \\
\hline & \multicolumn{3}{|c|}{$E T N$} & \multicolumn{3}{|c|}{$C M$} & \multicolumn{3}{|c|}{ SE } & \multicolumn{3}{|c|}{ SPE } \\
\hline & Peso & Superficie & $\%$ & Peso & Superficie & $\%$ & Peso & Superficie & $\%$ & Peso & Superficie & $\%$ \\
\hline P. engelmannii & 0.39 & 6200 & 31.0 & 0.18 & 3000 & 15.0 & 0.23 & 6200 & 31.0 & 0.26 & 6200 & 31.0 \\
\hline P. arizonica & 0.22 & 4500 & 22.5 & 0.22 & 4300 & 21.5 & 0.16 & 3000 & 15.0 & 0.12 & 2000 & 10.0 \\
\hline P. durangensis & 0.16 & 3300 & 16.5 & 0.40 & 6450 & 32.3 & 0.10 & 1800 & 9.0 & 0.16 & 3300 & 16.5 \\
\hline P. leiophylla & 0.11 & 2500 & 12.5 & 0.11 & 2750 & 13.8 & 0.22 & 4000 & 20.0 & 0.17 & 3500 & 17.5 \\
\hline P. chihuahuana & 0.07 & 2000 & 10.0 & 0.06 & 2000 & 10.0 & 0.13 & 2000 & 10.0 & 0.17 & 3500 & 17.5 \\
\hline P. cembroides & 0.05 & 1500 & 7.5 & 0.03 & 1500 & 7.5 & 0.16 & 3000 & 15.0 & 0.12 & 1500 & 7.5 \\
\hline Total & 1.00 & 20000 & 100.0 & 1.00 & 20000 & 100.0 & 1.00 & 20000 & 100.0 & 1.00 & 20000 & 1000 \\
\hline
\end{tabular}


En la figura 8 se muestra la distribución espacial óptima de las especies de interés considerando el escenario "calidad de la madera" (los expertos otorgaron una ponderación a las especies en función de la calidad de su madera). $P$. durangensis y $P$. ariz̧onica son las especies más adecuadas para el establecimiento de plantaciones en $32.3 \%$ y $21.5 \%$ del área disponible. Le sigue $P$. engelmannii, pudiendo ser plantada en $15 \%$ de la superficie disponible, P. leiophylla en $13.8 \%$ y P. chibuabuana en $10 \%$. Por otra parte, P. cembroides fue la especie con menor potencial óptimo con un $7.5 \%$ de la superficie (Tabla 4 ).

En el escenario "supervivencia de la especie", $P$. engelmannii, P. leiophylla, P. arizonica y P. cembroides resultaron ser las especies con mayor potencial (alto y medio), destacando la mencionada al inicio con 6200 ha; P. chibuabuana y $P$. durangensis presentaron un menor potencial óptimo con 10\% y 9\% respectivamente (Fig. 9, Tabla 4).

La distribución espacial óptima para el escenario "Susceptibilidad a plagas y enfermedades" se muestra en la figura $10 \mathrm{y}$ en la tabla 4 . Se observa nuevamente que $P$. engelmannii es la especie que podría ser plantada en un porcentaje mayor de superficie apta disponible (31\%), seguida de P. leiopbylla y P. chibuabuana, especies que podrían ser plantadas óptimamente en $17.5 \%$ de la superficie cada una. $P$. durangensis podría ser plantada en $16.5 \%$ del área y $P$. arizonica y $P$. cembroides en $10 \%$ y $7.5 \%$ del área respectivamente.

El AHP se utilizó en un ambiente espacial para evaluar la aptitud de terrenos para el establecimiento de plantaciones forestales comerciales con seis especies de pino en Durango, México. El proceso aplicado representa una propuesta de modelo de jerarquía que incorpora información de criterios climáticos, edafológicos y topográficos, y que puede considerarse una referencia para futuros estudios sobre aptitud de tierras para plantaciones forestales. El modelo fue construido teniendo en cuenta los elementos que la literatura científica y el aporte de técnicos forestales con experiencia en el establecimiento de plantaciones forestales comerciales. Su experiencia permitió identificar y ponderar los criterios relevantes para evaluar la aptitud de terrenos para establecer plantaciones. Por lo anterior, el modelo podría ser utilizado como punto de partida para generar modelos similares para otras especies arbóreas en otras latitudes, sin embargo, ni el modelo ni los criterios incluidos en él deben considerarse exhaustivos.

El AHP facilitó la tarea de calcular los valores de peso para cada uno de los criterios y subcriterios identificados como relevantes para la evaluación de la aptitud de los terrenos, un aspecto fundamental en el desarrollo de este tipo de modelos. El desarrollo del modelo de jerarquía y la ponderación de los criterios y subcriterios no son tareas triviales y deben realizarse con cuidado, AHP ha demostrado ampliamente su valor para realizar tales tareas (Bhushan y Rai, 2004; Brunelli, 2015).

A pesar de que AHP es una metodología de análisis de decisión madura, la aplicación aquí descrita es solo una realización de otras combinaciones posibles de métodos para estandarizar, métodos para ponderar criterios y métodos para ordenar alternativas de decisión (Malczewski, 1999). Existen otros enfoques y combinaciones de métodos para evaluar la aptitud de terrenos para establecer plantaciones forestales (Malczewski, 1999, 2004), siendo el más simple de estos el llamado análisis Booleano (DelgadoCaballero et al., 2010). Sin embargo, es preciso mencionar que, debido a su simplicidad, facilidad de uso y a su amplia fundamentación teórica, el AHP ha encontrado amplia aceptación entre los tomadores de decisiones (Brunelli, 2015) debido a que facilita la estructuración, el seguimiento y el análisis del problema de decisión. Adicionalmente, AHP ha demostrado ser una metodología capaz de producir resultados que concuerdan con las expectativas de los tomadores de decisiones (López-Upton, ValdezLazalde, Ventura-Ríos, Vargas-Hernández y Guerra-de-laCruz, 2015).

Respecto a los resultados del caso de estudio abordado, las diferencias en el área y la distribución espacial obtenida para cada especie, en función del escenario considerado, es atribuible a los valores de importancia definidos por los expertos para cada especie a través de las matrices de comparación pareada. Así, conforme a lo 
esperado, cuanto mayor es la ponderación para una especie en particular, más extensa resulta el área adecuada definida para esa especie en el mapa de distribución óptima.

A pesar de lo frecuente que resulta encontrar conflictos de uso del suelo al planear la realización de dos o más actividades no complementarias en un terreno forestal, particularmente en el establecimiento de plantaciones, los casos de evaluaciones multiobjetivo (MOLA) en el sector forestal de México son en extremo escasos, por lo que se dificulta realizar un análisis comparativo de la técnica utilizada. Martínez-Salvador y Prieto-Ruiz (2011) realizaron un análisis multicriterio Booleano para determinar áreas con potencialidad para establecer plantaciones forestales con tres especies de pino (Pinus arizonica P. durangensis y $P$. engelmannii) en todo el estado de Durango. Desafortunadamente su trabajo no muestra la distribución de potencialidad para cada una de las especies de manera separada, lo que dificulta su identificación con fines comparativos.

A diferencia del trabajo realizado por Pineda et al. (2012), en el que combinan las técnicas AHP, WLC y MOLA para localizar zonas de adecuación óptima para plantaciones forestales en el Estado de México con tres objetivos: comercial, de restauración y de protección y agroforestal, un aspecto innovador en esta investigación es el uso de una segunda aplicación de encuestas para generar matrices de comparación por pares que priorizan a las especies y con ello, la distribución espacial óptima para cada especie de pino bajo varios escenarios de decisión.

La mayoría de los estudios existentes en el tema no trascienden el análisis multicriterio realizado con enfoque Booleano, o mediante procedimientos de mayor complejidad como el AHP para definir la aptitud de tierras para un objetivo único (Bustillos-Herrera et al., 2007; Olivas-Gallegos et al., 2007; Delgado-Caballero et al., 2010; Martínez-Salvador y Prieto-Ruiz, 2011; Silva Gallegos et al., 2016; González-Ovando et al., 2016; Aguirre-Salado et al., 2017), es decir, solo abordan la Etapa 1 definida en este trabajo. Lo anterior, aunado a la carencia de mapas de escala grande (1:250000 o superior) que muestren con detalle la distribución natural de las especies objeto de este estudio, dificulta un comparativo de los resultados obtenidos. La opción de realizar recorridos de campo para corroborar la presencia de las especies en las áreas identificadas como óptimas desafortunadamente estuvo fuera del alcance presupuestario de la investigación. Sin embargo, es preciso mencionar que los mapas generados son concordantes espacialmente con los mapas de distribución de las especies bajo estudio generados por Perry (1991), y pueden ser útiles a tomadores de decisiones para enfocar esfuerzos locales de desarrollo de proyectos de plantaciones forestales maderables con fines comerciales.

\section{CONCLUSIONES}

El modelo jerárquico definido y aplicado conforme a la teoría del AHP, combinado con el procedimiento de análisis espacial multiobjetivo denominado MOLA, permitió definir la distribución espacial óptima de seis especies de Pinus con potencial para establecer plantaciones forestales comerciales y su posible distribución espacial, al considerar cuatro escenarios de decisión en el área de estudio. Adicionalmente, permitió determinar el grado de aptitud de la tierra para el establecimiento de plantaciones forestales comerciales con las especies de interés y localizar las áreas de conflicto entre especies. Los administradores de los recursos forestales del área de estudio e inversionistas pueden utilizar la información generada para enfocar sus programas de desarrollo de nuevas plantaciones forestales comerciales en la región. Por otro lado, el modelo jerárquico propuesto puede servir como referencia para la realización de estudios con objetivos similares en otras latitudes.

Los pesos asignados por los expertos a los criterios considerados, al igual que los considerados para los subcriterios, podrían resultar criticables por provenir de juicios emitidos por profesionales del ramo forestal considerados como expertos. Sin embargo, la toma de decisiones en cualquier área, particularmente en las que se carece de datos experimentales que demuestren la importancia de los elementos o variables consideradas en el 
proceso, tiene como opción el uso de este tipo de metodologías, a pesar de sus desventajas.

El municipio de Durango, Durango presentó la mayor cantidad de áreas óptimas para establecer plantaciones forestales con las especies analizadas. Al considerar el escenario "extensión del periodo de producción", se identificaron 6200 ha y 4500 ha de mejor aptitud para establecer plantaciones de $P$. engelmannii y $P$. arizonica, respectivamente. Con base en el escenario "calidad de la madera", $P$. durangensis y $P$. arizonica son las mejores especies a plantar con 6450 ha y 4300 ha, respectivamente. Pinus engelmannii y $P$. leiophylla son las especies con mejor potencial para el establecimiento de plantaciones si se considera el escenario "supervivencia de las especies" con 6200 ha y 4000 ha. Mientras que, P. engelmannii fue la especie más adecuada para establecer plantaciones en 6200 ha, al elegir el escenario "susceptibilidad a plagas y enfermedades".

\section{RECONOCIMIENTOS}

Se agradece de manera especial al Dr. José Ciro Hernández Díaz, al M.C. Ramón Silva Flores, a los Ings. Angélica Galindo Figueroa, Daniel Trujano Thomé y Roberto Trujillo por sus opiniones expertas para ponderar las especies para establecer plantaciones forestales.

\section{REFERENCIAS}

Aguirre-Salado, C. A., Valdez-Lazalde, J. R., Sánchez-Díaz, G., Miranda-Aragón, L., \& Aguirre-Salado, A.I. (2015). Modelling site selection for tree plantation establishment under different decision scenarios. Journal of Tropical Forest Science, 27(3), 298-313.

Aguirre-Salado, C. A., Miranda-Aragón, L., Pompa-García, M., Reyes-Hernández, H., Soubervielle-Montalvo, C., FloresCano, J. A., \& Méndez-Cortez, H. (2017). Improving identification of areas for ecological restoration for conservation by integrating USLE and MCDA in a GISenvironment: A pilot study in a priority region Northern Mexico. International Journal of Geo-Information, 6(9), 262. doi: 10.3390 /ijgi6090262

Bhushan, N. \& Rai, K. (2004). Strategic Decision Making: Applying the Analytic Hierarchy Process. London, UK: Springer.

Brunelli, M. (2015). Introduction to the Analytic Hierarchy Process. Nueva York, Estados Unidos: SpringerBriefs in Operations Research.
Bustillos-Herrera, J. A., Valdez-Lazalde, J. R., Aldrete, A., \& González-Guillén, M. J. (2007). Aptitud de terrenos para plantaciones de eucalipto: definición mediante el proceso de análisis jerarquizado y SIG. Agrociencia, 41(7), 787-796.

Comisión Nacional Forestal [Conafor]. (2013). Programa de Desarrollo de Plantaciones Forestales Comerciales: $A 15$ años de su creación. A. M. Fierros González (Ed.). Guadalajara, México: Semarnat-Conafor.

Delgado-Caballero, C. E., Valdez-Lazalde, J. R., FierrosGonzález, A. M., de los Santos-Posadas, H. M., \& GómezGuerrero, A. (2010). Aptitud de áreas para plantaciones de Eucalipto en Oaxaca y Veracruz: Proceso de análisis jerarquizado vs. Algebra booleana. Revista Méxicana de Ciencias Forestales, 1(1), 23-133.

Eastman, J. R. (2012). IDRISI ${ }^{\circledR}$ Selva. Guia para SIG y Procesamiento de Imágenes. Manual Versión 17. Worcester, Estados Unidos: Clark University.

Eastman, J. R., Jin, W., Kyem, P. A. K., \& Toledano, J. (1995). Raster procedures for multi-criteria/multi-objective decisions. Photogrammetric Engineering \& Remote Sensing, 61(5), 539-547.

Organización de las Naciones Unidas para la Agricultura y la Alimentación [FAO]. (2016). Evaluación de los recursos forestales mundiales 2015. ¿Cómo están cambiando los bosques del mundo? (2a ed.). Roma, Italia: FAO.

García-Arévalo, A. \& González-Elizondo, M. (2003). Pináceas de Durango (2a ed.). México, D.F.: Instituto de Ecología Comisión Nacional Forestal.

Gómez, D. M. \& Barredo, J. (2005). Sistemas de Información Geográfica y Evaluación Multicriterio en la Ordenación del Territorio (2a ed.). Madrid, España: Ra-Ma.

González-Ovando, M. L., Plascencia-Escalante, F. O., \& Martínez-Trinidad, T. (2016). Áreas prioritarias para restauración ecológica y sitios de referencia en la región Chignahuapan-Zacatlán. Madera y Bosques, 22(2), 41-52. doi: $10.21829 /$ myb.2016.2221323

Instituto Nacional de Estadística y Geografía [Inegi] (2005). Sistema de Descarga de Continuo de Elevaciones Mexicano. Recuperado de http:/ /www.inegi.gob.mx

López-Upton, J., Valdez-Lazalde, J. R., Ventura-Ríos, A., VargasHernández, J. J., \& Guerra-de-la-Cruz, V. (2015). Extinction Risk of Pseudotsuga Menziesii Populations in the Central Region of Mexico: An AHP Analysis. Forests, 6(5), 1598-1612. doi: 10.3390/f6051598

Malczewski, J. (1999). GIS and multicriteria decision analysis. Toronto, Canada: John Wiley and Sons. 
Malczewski, J. (2004). GIS-based land-use suitability analysis: A critical overview. Progress in Planning, 62, 3-65. doi: 10.1016/j.progress.2003.09.002

Malczewski, J. (2011). Local weighted linear combination. Transactions in GIS, 15(4), 439-455. doi: 10.1111/j.14679671.2011.01275.x

Martínez-Salvador, M. \& Prieto-Ruiz, J. A. (2011). Determinación de áreas potenciales para el establecimiento de plantaciones forestales comerciales en la Región Norte de México. Durango, México: INIFAP - Campo Experimental Valle del Guadiana.

Olivas-Gallegos, U. E., Valdez-Lazalde, J. R., Aldrete, A., González-Guillén, M. J., \& Vera-Castillo, G. (2007). Áreas con aptitud para establecer plantaciones de maguey cenizo: definición mediante análisis multicriterio y SIG. Fitotecnia Mexicana, 30(4), 411-419.

Payn, T., Carnus, J.-M., Freer-Smith, P., Kimberley, M., Kollert, W., Liu, S., Orazio, C., Rodriguez, L., Silva, L. N., \& Wingfield, M. J. (2015). Changes in planted forests and future global implications. Forest Ecology and Management, 352, 57-67. doi: 10.1016/j.foreco.2015.06.021

Perry, J. P. (1991). The pines of Mexico and Central America. Portland, Estados Unidos: Timber Press.

Pineda-Jaimes, N. B., Bosque-Sendra, J., Gómez-Delgado, M., Franco-Plata, R., Antonio-Némiga, X., \& Manzano-Solis, L. R. (2012). Determination of optimal zones for forest plantations in the State of Mexico using multi-criteria spatial analysis and GIS. Journal of Geographic Information System, 4(3), 204-218. doi: 10.4236/jgis.2012.43025

Saaty, T. L. (1980). The Analytic Hierarchy Process. Nueva York, Estados Unidos: McGraw-Hill.

Saaty, T. L. (1990). How to make a decision: The Analytic Hierarchy Process. European Journal of Operational Research, 48, 9-26. doi: 10.1016/0377-2217(90)90057-I
Santé-Riveira, I. \& Crecente-Maseda, R. (2005). Evaluación de métodos para la obtención de mapas continuos de aptitud para usos agroforestales. Geofocus: Revista Internacional de Ciencia y Tecnología de la Información Geográfica, 5, 40-68.

Secretaria del Medio Ambiente y Recursos Naturales - Colegio de Postgraduados [Semarnat-CP]. (2002). Evaluación de la degradación del suelo causada por el hombre en la República Mexicana Escala 1: 250.000. México, D.F.: Semarnat.

Silva-Gallegos, J. J., Aguirre-Salado, C. A., Miranda-Aragón, L., Sánchez-Díaz, G., Valdez-Lazalde, J. R., Pedroza-Carneiro, J. W., \& Flores-Cano, J. A. (2016). Locating Potential Zones for Cultivating Stevia rebaudiana In Mexico: Weighted Linear Combination Approach. Sugar Tech, 19(2), 206-218. doi:10.1007/s12355-016-0446-x

Valdez-Lazalde, J. R., López-Upton, J., \& Aldrete, A. (2003). Identificación de especies forestales para el establecimiento de plantaciones maderables y no maderables. Montecillo, México: Comisión Nacional Forestal - Colegio de Postgraduados.

Manuscrito recibido el 12 de julio de 2018

Aceptado el 28 de enero de 2019

Publicado el 1 de noviembre de 2019

Este documento se debe citar como:

Hernández-Zaragoza, P., Valdez-Lazalde, J. R., Aldrete, A., \& MartínezTrinidad, T. (2019). Evaluación multicriterio y multiobjetivo para optimizar la selección de áreas para establecer plantaciones forestales. Madera y Bosques, 25(2), e2521819. doi: 10.21829/myb.2019.2521819

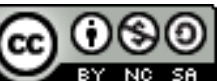

Madera y Bosques por Instituto de Ecología, A.C. se distribuye bajo una Licencia Creative Commons Atribución-NoComercialCompartirlgual 4.0 Internacional. 